\title{
'Patterning' frictional differentiation to a polymer surface by atomic force microscopy
}

\author{
Gregory S. Watson ${ }^{\mathrm{a}}$, Christopher L. Brown ${ }^{\mathrm{a}}$, Sverre Myhra ${ }^{\mathrm{b}}$, Nicolas C. Roch ${ }^{\mathrm{a}}$, Simon Hu ${ }^{\mathrm{a}}$, Jolanta \\ A. Watson ${ }^{\mathrm{a}}$
}

\author{
${ }^{a}$ Nanoscale Science and Technology Centre, School of Science, Griffith University; Nathan, \\ Queensland 4111, Australia \\ ${ }^{\mathrm{b}}$ Oxford University Begbroke Science Park, Yarnton OX5 1PF, UK
}

\begin{abstract}
The surface structure and chemistry of polymers affect their functionality for a great range of applications in areas as diverse as biosensors, corrosion protection, semiconductor processing, biofouling, tissue engineering and biomaterials technology. Some of those applications require purposeful tailoring of laterally differentiated regions (e.g., array structures for multi-channel/multi-analyte biosensors and patterning for promotion of selective adhesion of cells/proteins). While such tailoring is currently taking place on the $\mu \mathrm{m}$-scale, it is likely in the future to progress into the nm-regime. Attachment of biological moieties at surfaces and interfaces has been shown to be highly dependant on local chemistry at the intended site of attachment. Additionally, the local molecular-scale geometry may promote or hinder attachment events, as in the case of biofilms. To date, however, the effect of frictional properties of surfaces for chemical and biomolecular attachment is a much less understood phenomenon.

In this study we show controlled patterning of a polymer surface (polydimethylsiloxane (PDMS)) arising from manipulation by Atomic Force Microscopy (AFM). PDMS is a bio-active/selective polymer having a broad range of applications, such as biomedical devices, molecular stamps, hydraulic fluid devices and in soft lithography. The polymer surface has been selectively altered by high speed scanning in order to generate regions on the surface that exhibit differentiated frictional properties. By altering the loading force, scan width, and area of the AFM probe-to-polymer contact it is possible to produce a variety of detailed and complex patterns with frictional contrast, including anisotropic frictional gradients on the polymer surface. The controlled manipulation of the polymer surface can be carried out on the micro-, meso- and nano-scale.
\end{abstract}

Keywords: Atomic force microscopy, PDMS, Stick-Slip, Polymer, Friction, Manipulation

\section{INTRODUCTION}

Precise manipulation leading to laterally differentiated regions on polymeric materials is required for a range of applications/devices. Patterning surfaces will hinder or promote adhesion of various (bio)chemicals ${ }^{1-3}$. Ion beam sputtering, laser ablation, chemical etching and micro-ablation are just some of the well-established group of technologies collectively referred to as micro-machining. Scanning probe microscopy (SPM) has now become an addition to the variety of tools available. While some technologies such as chemical etching and ion beam sputtering rest on a firm scientific basis, the underlying science is much less satisfactory in the case of mechanical manipulation by SPM methods.

Current explanatory models for tip-induced manipulation of polymer surfaces are in their infancy. Earlier studies have generally been based on insight gained from unrelated materials ${ }^{4-9}$ with some studies investigating polymer materials scanned over reasonably small scan ranges $(\leq 5 \times 5 \mu \mathrm{m})$ with results showing polymer bundles forming as a result of action by the AFM probe ${ }^{10-14}$. The principal objective in this paper is to develop an understanding of tip-induced 
manipulation of a soft elastic polymer, Poly(dimethylsiloxane), (PDMS), with a focus on the directional response of the AFM probe during manipulation over 10 and $20 \mu \mathrm{m}^{2}$ scan areas.

PDMS is now routinely used in a wide range of patterning and bio-chemical isolation and biomedical applications ${ }^{15-19}$, and has a multitude of potential applications including insulation and anti-fouling coating $\mathrm{s}^{20-22}$. It offers a range of useful physical, mechanical and chemical properties including transparency, surface hydrophobicity, constant and high ductility over a wide range of temperatures, low toxicity, high electrical resistance, long-term stability and flexibility ${ }^{23}$. PDMS is also used in soft lithography, which is of particular relevance to this study. The fabrication and use of micro/nano stamps and fluidic channels using PDMS material has been demonstrated in a number of studies (e.g., [16, 24-26]). An understanding of the tribological properties and lithographic outcomes from AFM manipulation of PDMS surfaces is a necessary precursor for further technological exploitation.

\section{EXPERIMENTAL SECTION}

\subsection{Specimen materials}

PDMS (Sylgard ${ }^{\circledR}$-184) was supplied by Dow Corning as a two part silicone elastomer. The base and curing agent were mixed at a 10:1 weight ratio, spin-coated onto atomically flat silicon wafer substrates and cured in an ambient environment $\left(25^{\circ} \mathrm{C}\right.$ and $55 \%$ relative humidity) for 48 hours prior to any analysis or manipulation. The average thickness of the polymer was ca. $2 \mu \mathrm{m}$.

\subsection{AFM instrumentation}

The work was carried out on a ThermoMicroscope TMX-2000 Explorer, based on the detection of tip-to-surface forces through the monitoring of the optical deflection of a laser beam incident on a force-sensing/imposing lever. Scanning with the instrument is implemented by probe/lever being in motion while the stage assembly remains stationary. In order to cover the scales of lateral and topographical differentiation, a $130 \times 130 \mu \mathrm{m}^{2}$ tripod scanner (z-range of $\left.9.7 \mu \mathrm{m}\right)$ was used. The analyses were carried out under air-ambient conditions $\left(20-23^{\circ} \mathrm{C}\right.$ and $60-70 \%$ relative humidity).

\subsection{Probes}

The probe consists of a lever with an integral conical tip attached at its free end. The tip-to-surface contact area defines the interaction volume, whereby information such as topography, strength of in-plane and out-of-plane forces is extracted. The contact area also represents the point at which purposeful manipulation is effected. Many polymers can be considered as 'soft' objects. Therefore the imposition of forces at the point of contact will cause deformation and indentation, and an increase in contact area. Thus it is necessary to use levers with force constants, $k_{N}, \leq 0.1 \mathrm{nNnm}^{-1}$ in order to improve resolution and to avoid surface modification. Levers with $k_{N} \geq 4 \mathrm{nNnm}^{-1}$ enable surface manipulation.

The characteristics of probes employed in the present study are listed in table 1. Beam-shaped levers were used in order to ensure that only simple bending modes needed to be considered. The data for radius of curvature at the tip apex, $R_{\text {Tip }}$, aspect ratio (opening half angle) of the tip, $A_{r}$, and surface chemistry $\left(<10 \mathrm{~nm},<10^{\circ}\right.$ and native Si-oxide film, respectively) are summarized from the suppliers' specifications. The values of the force constant for normal deflection, $k_{N}$, of individual probes were determined from the resonance method described by Cleveland et al [27], and the torsional, $k_{T}$, and longitudinal, $k_{L}$, force constants were calculated from the standard expressions for a long and thin lever, described by Gibson et al [28].

Table 2. Probe specifications.

\begin{tabular}{cccc}
\hline Probe & $\boldsymbol{k}_{\boldsymbol{N}}\left(\mathrm{nNnm}^{-1}\right)$ & $\boldsymbol{k}_{\boldsymbol{T}}\left(\mathrm{nNnm}^{-1}\right)$ & $\boldsymbol{k}_{\mathbf{L}}\left(\mathrm{nNnm}^{-1}\right)$ \\
\hline A & 0.035 & 7.2 & 4.7 \\
B & 6.8 & 150 & 95 \\
C & 7.1 & 155 & 100 \\
D & 0.1 & 15 & 10 \\
E & 10.3 & 220 & 145 \\
F & 0.07 & 11 & 7.4 \\
G & 14 & 300 & 200 \\
\hline
\end{tabular}




\subsection{Imaging}

Under normal topographical imaging conditions, the 'over-scan' along the fast scan direction, is typically ca. $25 \%$, and is a general characteristic of SPM instruments. This over-scan feature is intended as a means of removing the static friction feature from images. Thus for $20 \times 20$ and $10 \times 10 \mu \mathrm{m}^{2}$ fields of view there were 4 and $2.56 \mu \mathrm{m}$ over-scans, respectively, in the fast scan direction. The over-scan must be taken into account in this study due to the manipulation taking place along the entire fast scan trace. The outcome was then imaged over a larger field of view with a soft lever. As a result the imaged areas are quoted as $24 \times 20 \mu \mathrm{m}^{2}$ and $12.56 \times 10 \mu \mathrm{m}^{2}$. Imaging of the manipulated regions on the polymer was carried out immediately after alteration of the surface.

\section{RESULTS AND DISCUSSION}

\subsection{Surface topography and tip indentation}

A contact mode topographical image of a freshly spun PDMS surface is shown in Figure 1(a) in 3-dimensional representation. In order to evaluate the polymer surface topography with minimal surface alteration the image was obtained with probe 'A' in table 1, with a force loading $<20 \mathrm{nN}$. Subsequent scanning of a larger field of view than the previously imaged region, revealed no discernible surface changes in characteristics/relief and no build up of displaced material at the scan edges. The surface roughness for a $1 \times 1 \mu \mathrm{m}^{2}$ region was $0.5 \pm 0.1 \mathrm{~nm}$.
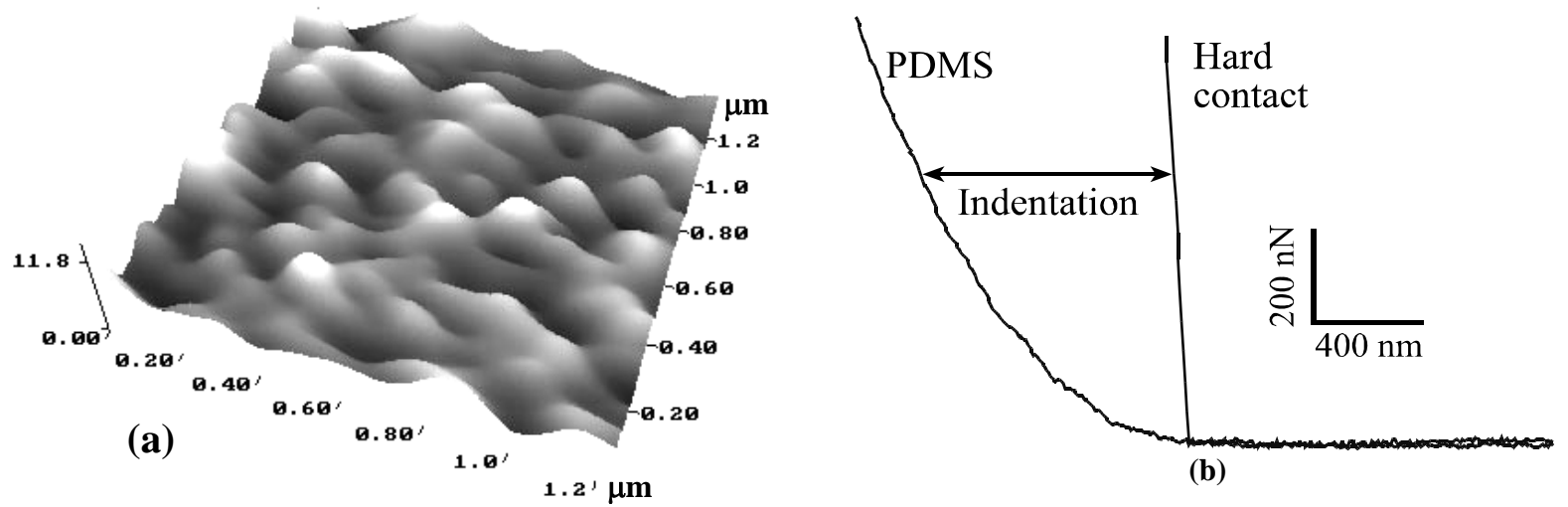

(b)

Figure 1. (a) Surface topography of a freshly spun PDMS surface shown in 3-D format. The contact mode image was obtained with probe 'A' at a force-loading of $<20 \mathrm{nN}$. (b) Representative $\mathrm{f}-\mathrm{d}$ curves obtained with probe ' $\mathrm{B}$ ' for an incompressible surface, polished $\mathrm{Si}$, and a PDMS surface. The latter curve shows the extent of tip indentation of the polymer. Tip penetration/indentation of ca. $950 \mathrm{~nm}$ is evident for an imposed force of ca. $600 \mathrm{nN}$. Subsequent imaging of the field of view after f-d analysis revealed total elastic recovery, within the resolution of imaging.

Figure 1(b) shows the approach half-cycles of two representative force versus distance (f-d) curves obtained on a 'hard' surface (clean silicon wafer) and a PDMS surface. The f-d data were obtained with probe 'B' (with a spring constant approximately two hundred times greater than probe ' $A$ '). The shape of the f-d curve taken on the PDMS surface shows significant out-of-plane deformation of the polymer. At a force of $600 \mathrm{nN}$, there was an indentation, $\Delta z$, of ca. $950 \mathrm{~nm}$. Using a model by Sneddon for deformation by a conical tip as a function of sample indentation ${ }^{29}$, Young's Modulus for the PDMS material was found to be in the range $3.1-7.6 \times 10^{5} \mathrm{~Pa}$. The results are in good agreement with the values quoted in the literature ${ }^{30-32}$.

\subsection{Stick-slip behaviour}

The PDMS surface was scanned at moderately high force loadings in order to evaluate the extent of polymer manipulation. The field of view was generated with a resolution of 300 lines. The velocity in the fast scan direction was $125 \mu \mathrm{ms}^{-1}$, and the raster over a field of $24 \times 20 \mu \mathrm{m}^{2}$ was carried out in the constant normal force mode (with probe ' $\mathrm{C}$ '). The applied normal force was $950 \mathrm{nN}$ and the fast scan direction $(x$-axis, $24 \mu \mathrm{m})$ was perpendicular to the long axis of the lever as shown in the inset in figure 2(a).

A lateral force image, shown in figure 2 (a), was obtained with a soft lever (probe 'A') which was used to scan after the manipulation of the PDMS surface using a stiff lever (probe ' $B$ '). The channel depth and width, as revealed by topographical images (not shown here), was found to be 160-260 nm and 0.4-0.6 $\mu \mathrm{m}$, respectively. The lateral force 
image clearly shows the series of horizontal channels and sloping lines connecting successive channels (arrows) showing the stick and slip mechanism, respectively, in the slow scan direction. The discontinuities/channels have an average spacing of approximately $2.3 \mu \mathrm{m}$. The location in the slow scan direction is incremented every two scanning lines in the fast scan direction in order to generate the raster pattern. Thus the scanning stage movement for a $20 \mu \mathrm{m}$ image (slow scan direction) incorporates $300 \times 2$ scan lines spaced $66.67 \mathrm{~nm}$ apart. Thus there will be ca. 69 scan lines for a $2.3 \mu \mathrm{m}$ stage-path along the slow scan direction, equal to the number of traverses per trough.
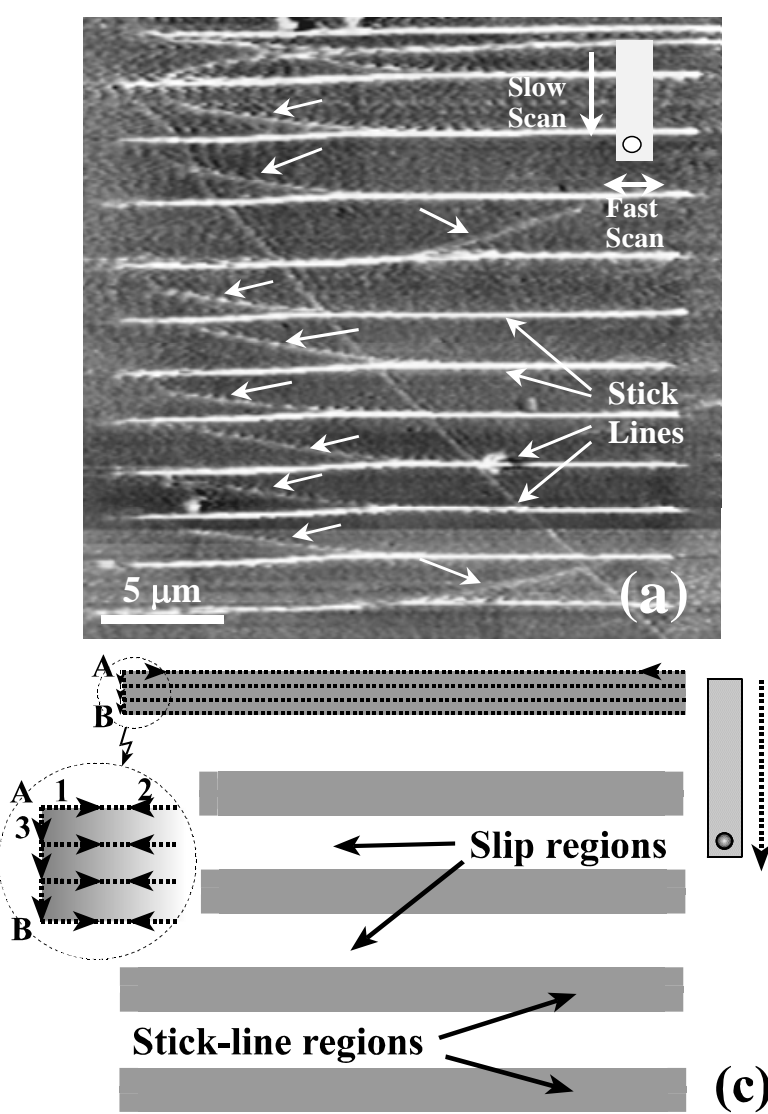
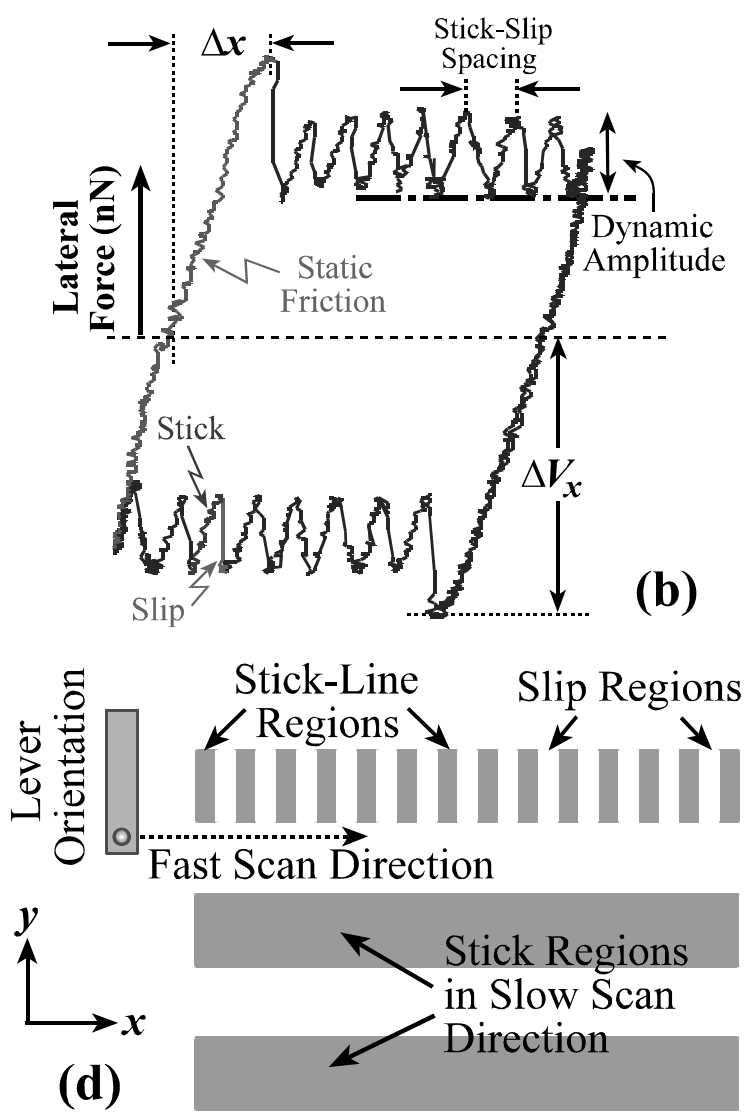

Figure 1. (a) Lateral force image of a manipulated region using a soft lever (probe ' $G$ ', table 1) showing the resulting stick lines and the path taken by the tip during the slip stage (arrows). (b) Friction loop obtained using probe ' $\mathrm{H}$ ' demonstrating stick-slip behaviour in the fast scan direction. $\Delta V_{x}$ represents the L-R detector signal with $\Delta x$ showing the lateral (in-plane) displacement of the stage. (c) $\mathrm{A}$ diagrammatic representation of the tip sticking, A to B (raster motion from 1 to 3 within a single grey band (channel) shown in the inset), then slipping (white band) through to the next grey region. (d) Schematic representation of the fast scan stick-slip motion of the lever.

Figure 2 (b) shows a representative friction loop acquired during the middle of a manipulating scanning cycle within a stick line in the fast scan direction (probe ' $\mathrm{C}$ ', table 1). The static friction is the regime in which the motion of the tip and sample are coupled. The amplitude and spacing of the stick-slip features are also defined. The friction loop clearly reveals stick-slip behaviour in the fast scan direction during the acquisition of an image and manipulation of the polymer. Figure 2(c) shows a diagrammatic representation of the manipulation event. The tip begins its raster motion at point $\mathrm{A}$ moving towards point B. This is illustrated in the inset whereby the raster motion proceeds from 1 to 3 within a single grey band (channel). The tip becomes trapped within the grey horizontal bands which represents one full stick cycle. As a consequence, only a small spatial region along the slow scan direction is imaged. Significant polymer deformation is evident during this process due to the stick point being dragged along in response to the longitudinal/buckling force imposed by the lever. Figure 2 (d) shows a schematic representation of the stick-slip behaviour in the fast scan direction similar to that shown in figure 2 (c) for the stick-slip process in the slow scan direction. As the tip moves across the surface ( $x$-direction) it becomes embedded at the first stick point until a point of instability is reached between the 
restoring force of the deformed polymer and the torsional force of the tip. This instability causes the tip to be released thereby jumping to its next stick position, repeating the cycle in both the forward and reverse directions of travel.

\subsection{Loading Force Dependence}

An investigation of loading force dependence on lateral force, channel spacing and depth was conducted. Figure 3 (a) shows a linear dependence of the lateral force on various force loadings using a stiff lever (probe ' $D$ '). At higher force loadings the elastic limit of a softer surface will be exceeded thus resulting in plastic deformation. Trends between loading force and depth (right axis) and spacing (left axis) is shown in (b). The depth and channel spacing increases with loading force due to the tip being embedded deeper into the surface, causing an increase in contact area, greater trapping and elastic deformation of the polymer (both in the $\mathrm{x}$ and $\mathrm{y}$ directions) The greater in-plane elastic force causes an increase in the spacing.
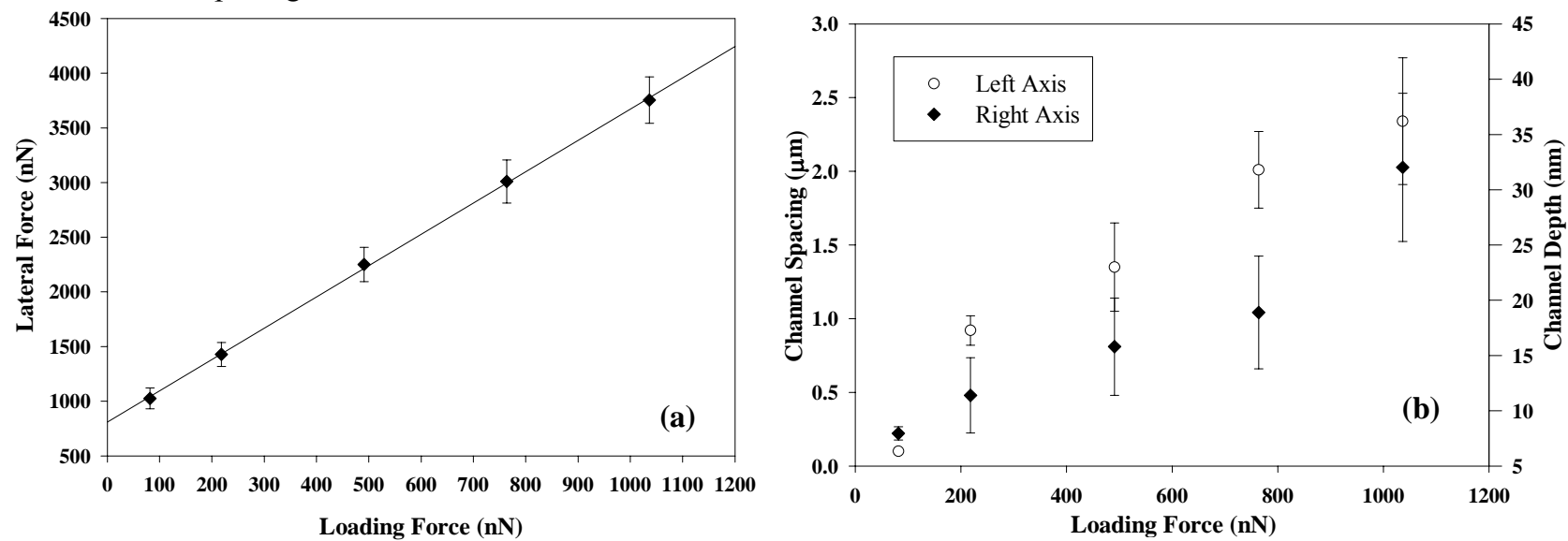

Figure 3. Dependence of lateral force $(R=0.999)$ (a), channel depth $(R=0.963)$ (right axis) and spacing between successive channels (left axis) (b), on loading force using probe ' $\mathrm{D}$ ' defined in table 1.

\subsection{Scan Speed Dependence}

A study of the dependence of scan speed on loading force, dynamic stick-slip amplitude (defined in figure 2 (b)), number of stick-slip features and their spacing has also been undertaken. Figure 4 (a) shows representative data demonstrating a clear dependence of lateral force on the scan speed in the fast scan direction (left axis). As the speed increases, there is an increase in contact between the sides of the polymer surface and the leading face of the tip, resulting in an increase in lateral force in the slow scan direction. Also, the probe may be increasingly restrained by the polymer. A similar trend occurs in the fast scan direction, whereby the dynamic stick-slip amplitude (right axis in figure 4 (a)) increases. The representative data presented in (b) shows a decrease in the number of stick-slip features (right axis) and an increase in the spacing between them (defined in figure 2 (b)) (left axis).
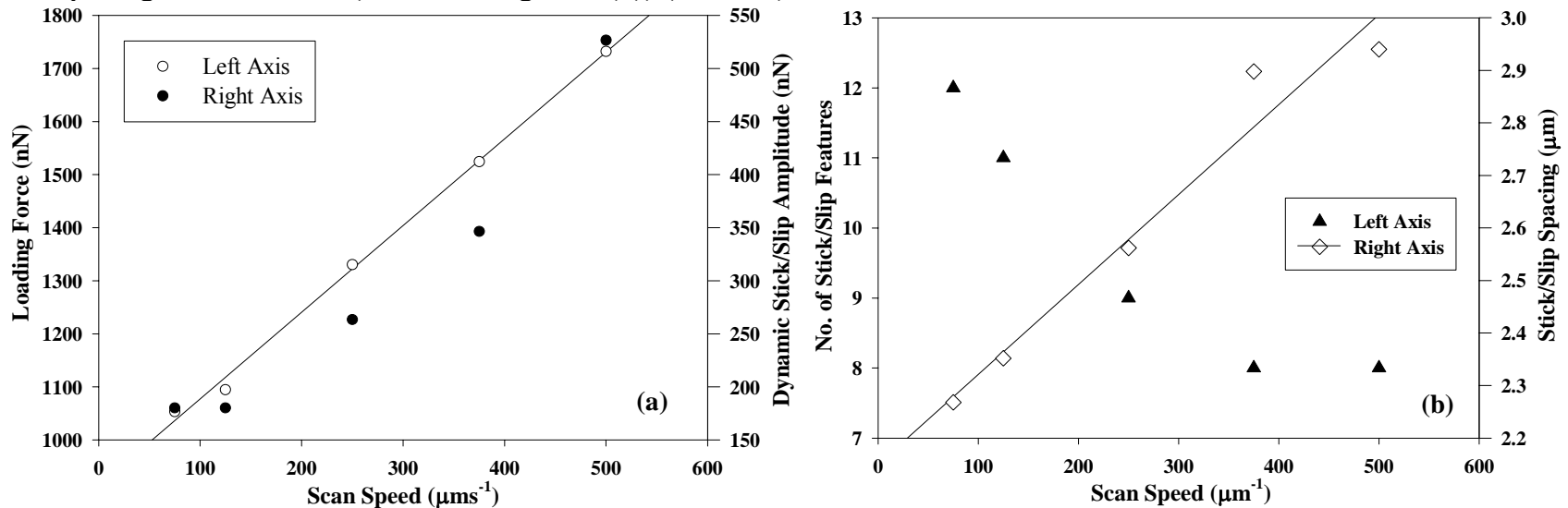

Figure 4. (a) Graphs showing a clear lateral force $(R=0.997)$ (left axis) and dynamic stick-slip amplitude dependence (right axis) on the increase in scan speed. (b) Dependence of the number of stick/slip features (right axis) and their spacing (left axis) on scan speed $(R=0.957)$. Data obtained using probe 'A' in table 1 . 


\subsection{Image Resolution Dependence}

The dependence of the number of channels, spacing and depth on image resolution has also been studied. Table 2 below indicates from experiments that the higher the resolution, the greater the number of times the tip traverses within a single channel before slipping to the next stick point.

Table 2. Spacing between consecutive rasters (one raster $=$ forward and reverse), and number of rasters per channel $(\times 2$ for the forward and reverse motion) for $10 \times 10 \mu \mathrm{m}^{2}$ image

\begin{tabular}{ccc}
\hline Resolution & One raster spacing $(\mathbf{n m})$ & \# of rasters / trough $(\times \mathbf{2})$ \\
\hline $50 \times 50$ & 200 & 6 \\
$100 \times 100$ & 100 & 22 \\
$300 \times 300$ & 33 & 132 \\
$400 \times 400$ & 25 & 156 \\
$500 \times 500$ & 20 & 196 \\
\hline
\end{tabular}

Figure 5 shows the general trends observed as the resolution was gradually increased, using probe ' $E$ ' and ' $\mathrm{B}$ ' during and after manipulation, respectively. The loading force of $465 \mathrm{nN}$ during manipulation, scan speed of $125 \mu \mathrm{ms}^{-1}$ and field of view of $10 \times 10 \mu \mathrm{m}^{2}$ were all kept constant for consistency. The results show an increase in channel spacing and depth after a resolution of 100 lines.

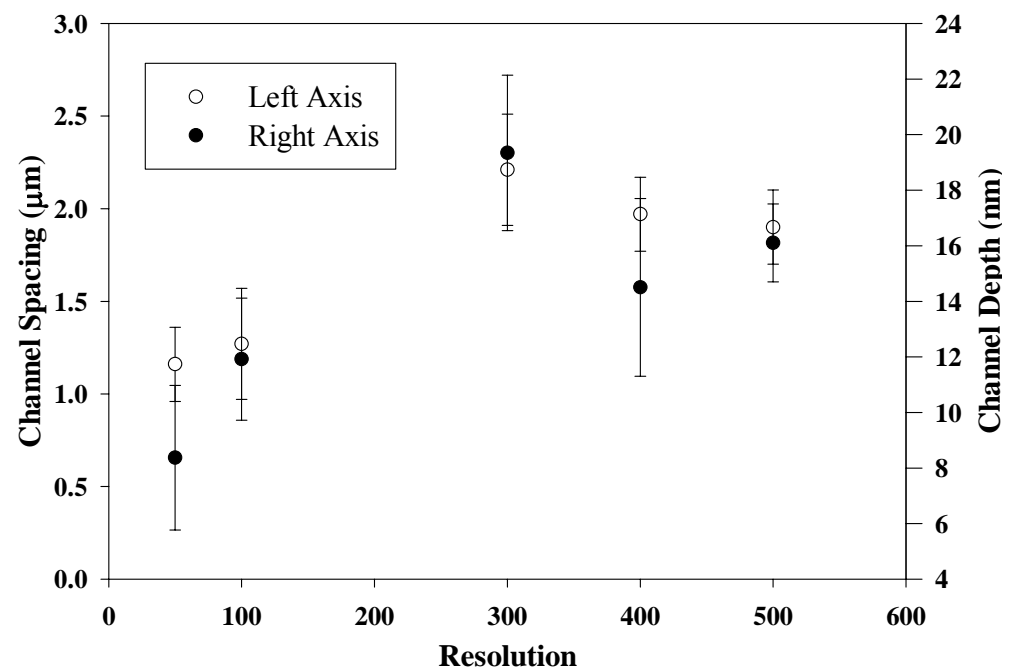

Figure 5. Representative graph showing the spacing between channels (left axis) and their depth (right axis) increasing with an increase in image resolution.

Figure 6 shows the general features of the stick-slip regime for high and low resolution image lithography. At a low resolution (e.g., $50 \times 50$ or $100 \times 100$ lines), the incremental movement of the tip in the $y$-direction is greater (200 nm at $50 \times 50$ resolution) than that at a high resolution $(66 \mathrm{~nm}$ at $300 \times 300$ resolution). This results in a lower number of rasters within a channel allowing the tip apex to be removed from the stick region. As the resolution increases, the number of rasters within a trough increases allowing the tip apex to become embedded further into the polymer surface, trapping the tip more efficiently, and providing a stronger restraining stick region. This results in the reduction in the number of troughs created over a scan area. 


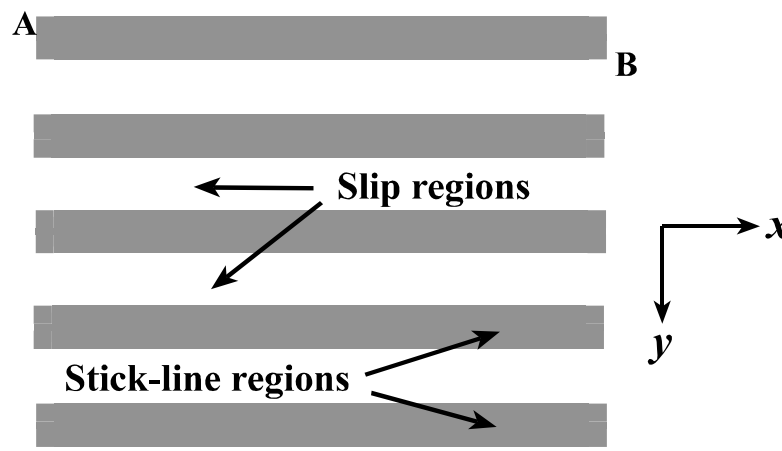

High resolution

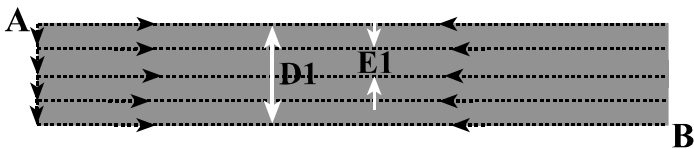

B
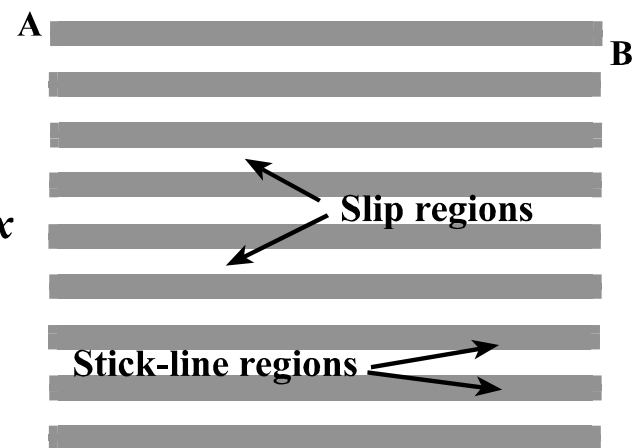

\section{Low resolution}

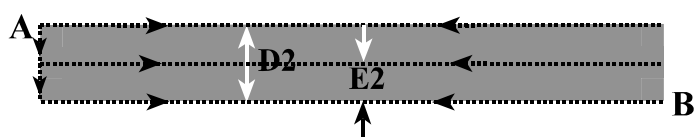

Figure 5. Schematic representation of the general stick-slip features for high and low resolution image lithography. At a high resolution, the raster increment, E1, is lower than the raster increment, E2, at a low resolution. The stick region is also greater at a high resolution, that is, D1 > D2.

\subsection{Homogeneous frictional architecture}

By altering the loading force it is possible to induce or prevent the stick-slip phenomenon. Figure 6 (a) shows a friction force image of a manipulated region on the PDMS surface. The manipulation was carried out at high force loading (ca. $600 \mathrm{nN}$ ) in order to induce stick-slip on the polymer surface, and thus create evenly spaced channels, as previously described in sections 3.2 - 3.5. Figure 6 (b) shows a friction force image resulting from a lower force loading of ca. 200 $\mathrm{nN}$. The outcome is a uniform, laterally differentiated region. The two distinctly different outcomes shown in figure 6(a) and (b) demonstrate the ability to form homogeneous and inhomogeneous frictional surface profiles using manipulative atomic force microscopy.
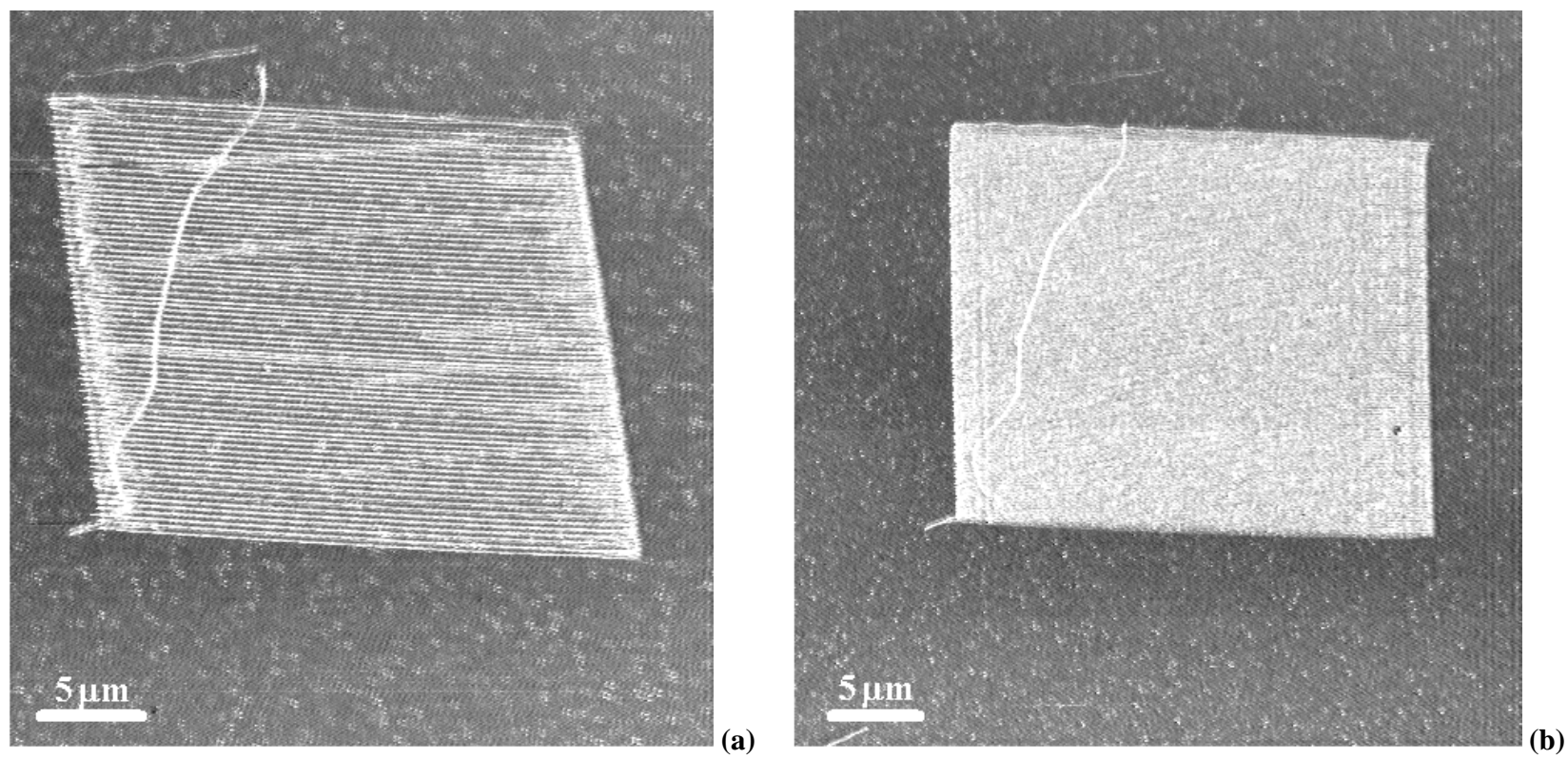

Figure 6 - (a) Friction force image of a manipulated region using a force loading of ca. $600 \mathrm{nN}$ resulting in uniformly spaced channels due to stick-slip. (b) Friction force image of a manipulated region using a lower force loading (ca. $200 \mathrm{nN}$ ) showing a uniform laterally differentiated region. 
By altering the scan angle, it is possible to produce features with various orientations. Figure 7 shows a topographical (a) and a friction force map (b) showing details of channels with sub- $\mu \mathrm{m}$ spacing created as a result of the stick-slip phenomenon. The channels were scanned $45^{\circ}$ to each other, thus creating precise mesh-like patterns. The line profile in (c), corresponding to the image in (a), shows an average depth of the channels to be ca. $15 \mathrm{~nm}$.



Figure 7 - (a) Topographical and (b) lateral force images of two overlapping areas showing details of channels created at varying orientations. The line profile in (c), corresponding to the topographical image in (a), shows the depth of channels to be ca. $15 \mathrm{~nm}$. The frictional force image (b) shows a higher frictional force within the channels.

Frictional force images in figure 8 show three manipulated regions within a single field of view, with the corresponding friction loops revealing higher friction on the manipulated regions. Squares A and B are the result of three and four repetitive rasters, respectively, using a high spring constant lever. The corresponding friction loops show the difference in friction in the two squares, i.e., $\Delta F_{F}$ of square $\mathrm{A}$ is lower than that of square $\mathrm{B}$. Square $\mathrm{C}$ is the result of a single raster over a $20 \times 20 \mu \mathrm{m}^{2}$ area, with a subsequent raster over a $10 \times 10 \mu \mathrm{m}^{2}$ area (square D), creating a frictional 'tier'. The RMS surface roughness of the manipulated region on square B was found to be higher (ca $2.8 \mathrm{~nm}$ ) than that of the surrounding PDMS surface (ca $1.8 \mathrm{~nm}$ ), for a $4 \times 4 \mu \mathrm{m}^{2}$ area. 

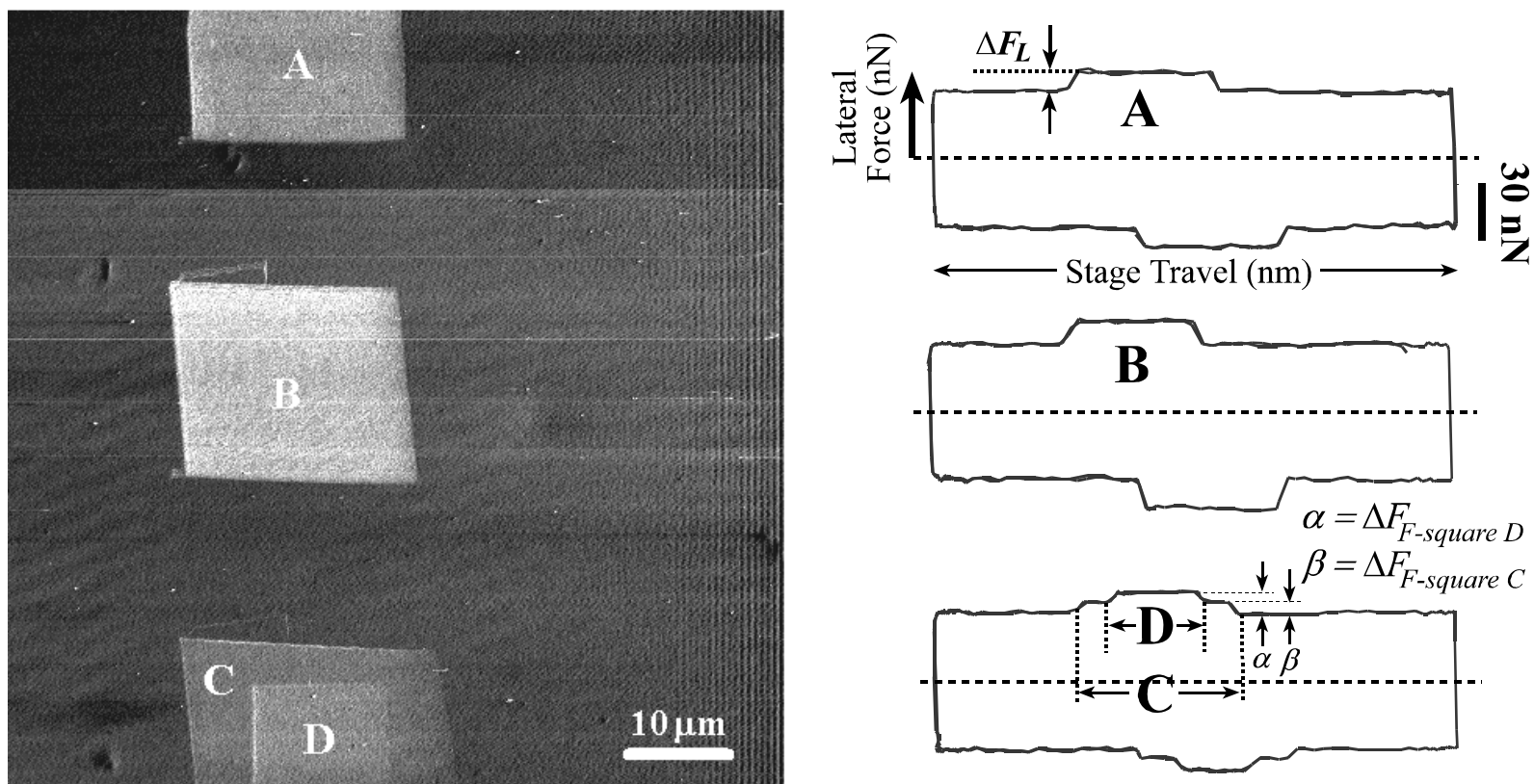

Figure 8 - A frictional force image (left) showing four square regions shows the dependence on the number of successive rasters; three for square A, four for square B, and one for square C. Square D is a result of an additional raster over square C once. The corresponding friction loops (right) demonstrate that friction increased with the number of repetitive rasters. The slight distortion in the images is due to thermal drift.

Figure 9 shows a variety of frictional architectures created on the PDMS surface by altering the loading force, scan size and the number of successive scans. The image shows overlapping of both homogeneous and inhomogeneous features. Squares A, B, D and E are created by maintaining a force loading below the point at which stick-slip is induced, thereby creating uniformly frictionally altered regions. Several squares overlap creating 'tiers' of changing friction, e.g., squares A, B and C. Stick-slip effects are apparent on square C.

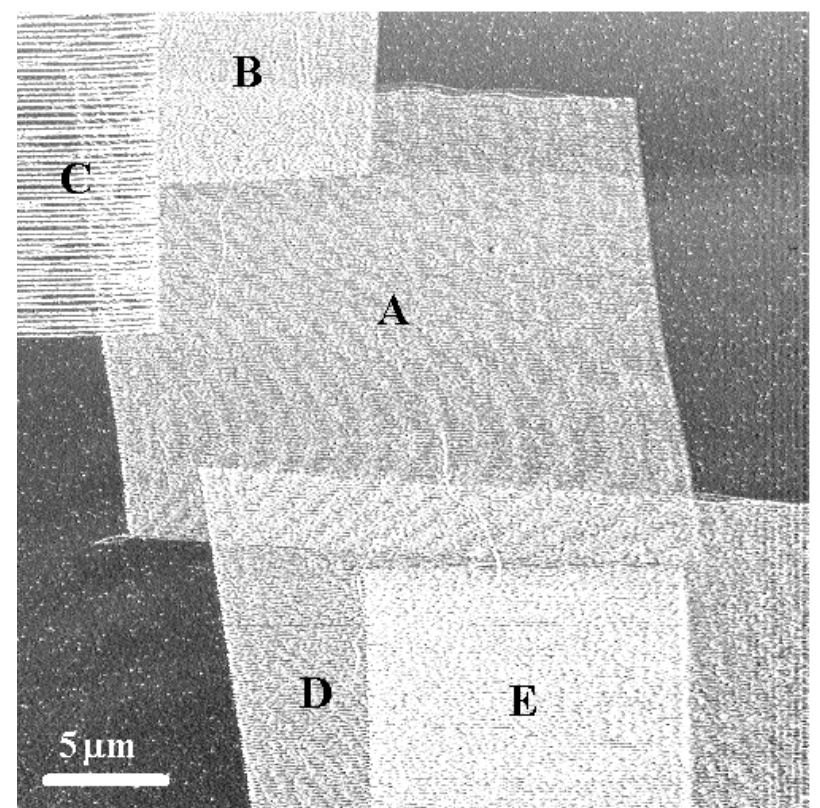

Figure 9 - Frictional force images showing the degree of freedom in feature formation on the polymer surface. 


\section{CONCLUSION}

Stick-slip effects have been observed in previous AFM studies; these have, in most cases, been restricted to atomic and molecular scales ${ }^{33-35}$. In the case of AFM-based manipulation of polymers the focus has generally been on establishing the dependence on loading force ${ }^{1,6,10,36,37}$, and/or temperature and number of rasters ${ }^{12-14}$. The present results for PDMS show, in particular, friction loop acquisition and analysis during manipulation, and describe in some detail the response of the AFM probe when it is in dynamic contact with a soft elastic polymer surface.

Surface alteration has been correlated with the response of the probe to linear motion and to lateral forces imposed by the relaxing polymer. An explanatory model was constructed with the aid of friction loop analysis, whereby the tip not only sticks and slips within the trough, in the fast scan direction, but also from one trough to another, in the slow scan direction. The process was consistently reproduced with levers with a spring constant of $>1 \mathrm{~N} / \mathrm{m}$. The in-plane relaxation of the surface in response to tip-induced in-plane forces, dynamic stick-slip amplitude and stick-slip spacings have also been examined.

By altering the scan conditions and probe parameters it is possible to carry out frictional patterning on a polymer surface (in this case PDMS) at the micro and nano scales. The frictional patterning can be carried out to form intricate frictional profiles. The pattered surfaces may have applications in regard to selective adsorption and separation of biological molecules.

\section{ACKNOWLEDGEMENTS}

We would like to acknowledge Ray Sweatman for his assistance with spin-coating and with supplying the PDMS materials.

\section{REFERENCES}

1. Blach JA, Watson GS, Brown CL, Pham DK, Wright J, Nicolau DV, Myhra S, "A mechanistic approach to tipinduced nano-lithography of polymer surfaces", Thin Solid Films, 459, 95-99, 2004.

2. Blach-Watson JA, Watson GS, Brown CL, Myhra S, "UV patterning of polyimide: differentiation and characterization of surface chemistry and structure" Applied Surface Science, 235, 164-169, 2004.

3. Nicolau DV, Taguchi T, Taniguchi H, Yoshikawa S, "Negative and positive tone protein patterning on Ebeam/deep-UV resists", Langmuir, 15, 3845-3851, 1999.

4. Hector LG, Schmid SR, "Simulation of asperity plowing in an atomic force microscope Part I: Experimental and theoretical methods", Wear, 215, 247-256, 1998.

5. Schmid SR, Hector LG, "Simulation of asperity plowing in an atomic force microscope. Part II: Plowing of aluminium Alloys", Wear, 215, 257-266, 1998.

6. Fang T-H, Went C-I, Chang J-G, "Machining characterization of the nano-lithography process using atomic force microscopy", Nanotechnology, 11, 181-187, 2000.

7. Bhushan B, "Nano- to microscale wear and mechanical characterization using scanning probe microscopy", Wear, 251, 1105-1123, 2001.

8. Pickering JP, Vancso GJ, "On the formation of oriented nanometer scale patterns on amorphous polymer surfaces studied by atomic force microscopy", Applied Surface Science, 148, 147-154, 1999.

9. Capella B, Sturm H, Weidner SM, "Breaking polymer chains by dynamic plowing lithography", Polymer, 43, 4461-4466, 2002.

10. Elkaakour Z, Aime JP, Bouhacina T, Odin C, Masuda T, "Bundle formation of polymers with atomic force microscope in contact mode: A friction versus peeling process", Physical Review Letters, 73, 3231-3234, 1994.

11. Haugstad G, Gladfelter WL, Jones RR, "Scanning force microscopy characterization of viscoelastic deformations induced by precontact attraction in a low cross-link density gelatin film", Langmuir, 14, 3944-3953, 1998.

12. Schmidt RH, Haugstad G, Gladfelter WL, "Correlation of Nanowear patterns to viscoelastic response in a thin polystyrene melt", Langmuir, 15, 317-321, 1999.

13. Schmidt RH, Haugstad G, Gladfelter WL, "Scan-induced patterning in glassy polymer films: Using scanning force microscopy to study plastic deformation at the nanometer length scale", Langmuir, 19, 898-909, 2003. 
14. Schmidt RH, Haugstad G, Gladfelter WL, "Scan-induced patterning and the glass transition in polymer films: Temperature and rate dependence of plastic deformation at the nanometer length scale", Langmuir, 19, 1039010398, 2003.

15. Xia Y, Whitesides GM, "Soft lithography", Angewandte Chemie International Edition, 37, 550-575, 1998.

16. Kane RS, Takayama S, Ostuni E, Ingber DE, Whitesides GM, "Patterning proteins and cells using soft lithography", Biomaterials, 20, 2363-2376, 1999.

17. Brinkmann M, Chan V Z-H, Homas EL, Lee VY, Miller RD, Hadjichristidis N, Avgeropoulos A, "Roomtemperature synthesis of $a-\mathrm{SiO}_{2}$ thin films by UV-assisted ozonolysis of a polymer precursor", Chemical Materials, 13, 967- 972, 2001.

18. Schnyder B, Lippert T, Kotz R, Wokaun A, Graubner V-M, Nuyken O, "UV-irradiation induced modification of PDMS films investigated by XPS and Spectroscopic Ellipsometry", Surface Science, 532-535, 1067-1071, 2003.

19. Ikada Y, "Surface modification of polymers for medical applications", Biomaterials, 15, 725-736, 1994.

20. Kim J, Chaudhury MK, Owen MJ, "Hydrophobicity loss and recovery of silicone HV insulation", IEEE Transactions on Dielectric and Electrical Insulation, 6, 695-702, 1999.

21. Hillborg H, Gedde UW, "Hydrophobicity changes in silicone rubbers", IEEE Transactions on Dielectric and Electrical Insulation, 6, 703-717, 1999.

22. Pike JK, Ho T, Wynne K, "Water-induced surface rearrangements of Poly(dimethylsiloxane-urea-urethane) segmented block copolymers", Chemistry of Materials, 8, 856-860, 1996.

23. Olah A, Hillborg H, Vancso GJ, "Hydrophobic recovery of UV/ozone treated poly(dimethylsiloxane): Adhesion studies by contact mechanics and mechanism of surface modification", Applied Surface Science, 239, 410-423, 2005.

24. Hu J, Deng T, Beck RG, Westervelt RM, Whitesides GM, "Fabrication of arrays of Schottky diodes using microtransfer molding", Sensors \& Actuators A, 75, 65-69, 1999.

25. Schueller OJA, Brittain ST, Whitesides GM, "Fabrication of glassy carbon microstructures by soft lithography", Sensors \& Actuators A, 72, 125-139, 1999.

26. Deng T, Prentiss M, Whitesides GM, "Fabrication of magnetic microfiltration systems using soft lithography", Applied Physics Letters, 80, 461-463, 2002.

27. Cleveland JP, Manne S, Bocek D, Hansma PK, "A nondestructive method for determining the spring constant of cantilevers for scanning force microscopy”, Review of Scientific Instruments, 64, 403-405, 1993.

28. Gibson CT, Watson GS, Myhra S, "Scanning force microscopy - calibration procedures for "best practice", Scanning, 19, 564-581, 1997.

29. Sneddon N, "The relation between load and penetration in the axisymmetric boussinesq problem for a punch of arbitrary profile", International Journal of Engineering Science, 3, 47-57, 1965.

30. Armani D, Liu C, Aluru N, $12^{\text {th }}$ Int. Conf. on MEMS (Orlando Florida) 222, 1998.

31. McDonald JC, Whitesides GM, "Poly(dimethylsiloxane) as a material for fabrication microfluidic devices", Accounts of Chemical Research, 35, 491-499, 2002.

32. Roca-Cusachs P, Rico F, Martinez E, Toset J, Farre R, Navajas D, "Stability of microfabricated high aspect ratio structures in Poly(dimethylsiloxane)", Langmuir, 21, 5542-5548, 2005.

33. Watson GS, Dinte BP, Blach-Watson JA, Myhra S, "Friction measurements using force versus distance friction loops in force microscopy", Applied Surface Science, 235, 38-42, 2004.

34. Mate CM, McClelland GM, Erlandsson R, Chiang S, "Atomic-scale friction of a tungsten tip on a graphite surface”, Physical Review Letters, 59, 1942-1945, 1987.

35. van den Oetelaar RJA, Flipse CFJ, "Atomic-scale friction on diamond (111) studied by ultra-high vacuum atomic force microscopy", Surface Science Letters, 384, L828-L835, 1997.

36. Garnaes J, Bjornholm T, Zasadzinski JAN, "Nanoscale lithography on langmuir-blodgett films of behinic acid", Journal of Vacuum Science and Technology B, 12, 1839-1842, 1994.

37. Ling JSG, Leggett GJ, Murray AJ, "Scanning force microscopy of polyester films: Contact versus non-contact imaging and tip-induced wear experiments", Polymer, 39, 5913-5921, 1998. 